\title{
El desempeño docente desde la perspectiva de la práctica profesional
}

Teaching Performance from the Perspective of Professional Practice

Desempenho docente na perspectiva da prática profissional

Guadalupe Iván Martínez Chairez

Escuela Normal Rural Ricardo Flores Magón, México ivan.martinez@enrrfm.edu.mx https://orcid.org/0000-0001-7925-6305

Ana Yocihra Esparza Chávez

Universidad Pedagógica Nacional del Estado de Chihuahua, México anaesparzachavez@gmail.com https://orcid.org/0000-0001-7238-8612

Rosa Isela Gómez Castillo Escuela Normal Rural Ricardo Flores Magón, México rosygomez@mac.com https://orcid.org/0000-0002-6891-8526 


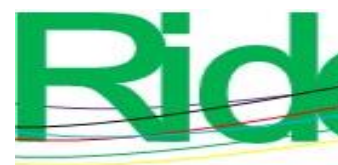
Revista Iberoamericana para la
Investigación y el Desarrollo Educativo
ISSN $2007-7467$

\section{Resumen}

El presente estudio se desarrolló en la región centro sur del estado de Chihuahua, en el Sector Educativo 25, el cual está integrado por cinco zonas escolares. La investigación se realizó mediante un paradigma pragmático con un enfoque mixto y un diseño secuencial exploratorio correlacional. En este documento se exponen los resultados de la fase cuantitativa y el contraste de las dos fases del estudio, con base en el objetivo de conocer la relación entre la evaluación del desempeño docente en su práctica y la evaluación docente a través de una prueba estandarizada. Algunos de los resultados son que $2.4 \%$ de la muestra representativa presentó un excelente desempeño docente, $7.3 \%$ un mal desempeño docente y $39 \%$ de los docentes observados registró un buen desempeño docente. Existe una relación -0.416 con una significancia menor 0.05 entre los años de estar trabajando en la misma escuela y el desempeño docente; no existe asociación entre el grado de estudios del profesor y el desempeño docente; además, se encontró que la correlación entre el desempeño docente en la práctica profesional y el desempeño docente evaluado a través de una prueba estandarizada es de 0.099, pero con una significancia de 0.686 , por ende, en esta investigación se establece que no existe relación. Y se concluye que es necesario una evaluación del desempeño docente dentro del aula, la cual refleje un panorama real de la práctica del profesorado, además de proporcionarle una retroalimentación para mejorar las áreas de oportunidad detectadas, debido a que un examen no refleja el desempeño docente contextualizado a las situaciones que se presentan en el actuar diario.

Palabras clave: desempeño docente, evaluación docente, modelos de evaluación docente, práctica reflexiva, prueba estandarizada. 


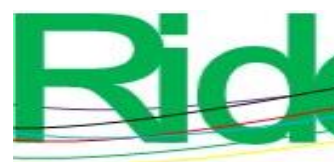

Revista Iberoamericana para la Investigación y el Desarrollo Educativo ISSN 2007-7467

\section{Abstract}

The present study was developed in the central south region of the state of Chihuahua, in Educational Sector 25, which is made up of five school zones. The research was carried out through a pragmatic paradigm with a mixed approach with correlational sequential exploratory design. This document presents the results of the quantitative phase and the contrast of the two phases of the study, based on the objective of knowing the relationship between the evaluation of teacher performance in their practice and the teacher evaluation through a standardized test. Some of the results are: $2.4 \%$ of the representative sample presented excellent teaching performance, $7.3 \%$ poor teaching performance, and $39 \%$ of teachers observed presented good teaching performance. There is a -0.416 relationship with a significance of less than 0.05 between the years of working in the same school and the teaching performance; there is no association between the degree of studies of the teacher and their teaching performance; it was also found that the correlation between teacher performance in professional practice and teacher performance evaluated through a standardized test is 0.099 , but with a significance of 0.686 , therefore, in this research it is established that there is no relationship. Therefore, it is concluded that an evaluation of the teaching performance within the classroom is necessary, which reflects a real panorama of the practice of the teaching staff, in addition to providing a feedback to improve the areas of opportunity detected, because an exam does not reflect the contextualized teaching performance to the situations that occur in daily acting.

Keywords: teaching performance, teacher evaluation, models of teacher evaluation, reflective practice, standardized test. 


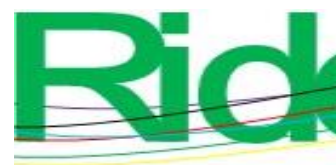
Revista Iberoamericana para la
Investigación y el Desarrollo Educativo
ISSN $2007-7467$

\section{Resumo}

Este estudo foi realizado na região centro-sul do estado de Chihuahua, no setor educacional 25, formado por cinco zonas escolares. A pesquisa foi realizada utilizando um paradigma pragmático, com abordagem mista e desenho seqüencial exploratório correlacional. Neste documento, são apresentados os resultados da fase quantitativa e o contraste das duas fases do estudo, com base no objetivo de conhecer a relação entre a avaliação do desempenho do ensino em sua prática e a avaliação do ensino através de um teste padronizado. Alguns dos resultados são que $2,4 \%$ da amostra representativa apresentou um excelente desempenho docente, 7,3\% um fraco desempenho docente e 39\% dos professores observados registraram um bom desempenho docente. Existe uma relação -0,416 com significância inferior a 0,05 entre os anos de trabalho na mesma escola e o desempenho docente; não há associação entre o grau de instrução e o desempenho do professor; Além disso, verificou-se que a correlação entre desempenho docente na prática profissional e desempenho docente avaliado por teste padronizado é de 0,099, mas com significância de 0,686, portanto, esta pesquisa estabelece que não há relação. E conclui-se que é necessária uma avaliação do desempenho do ensino em sala de aula, o que reflete um panorama real da prática de ensino, além de fornecer feedback para melhorar as áreas de oportunidade detectadas, porque um exame não reflete o desempenho do ensino contextualizado às situações que surgem na ação diária.

Palavras-chave: desempenho do professor, avaliação do professor, modelos de avaliação do professor, prática reflexiva, teste padronizado.

Fecha Recepción: Febrero 2020

Fecha Aceptación: Julio 2020 


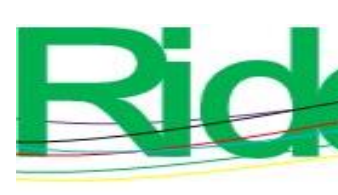

Revista Iberoamericana para la Investigación y el Desarrollo Educativo ISSN 2007-7467

\section{Introducción}

Desde la década de los 90 el Gobierno mexicano ha considerado a la evaluación docente como una herramienta estratégica para la mejora del sistema educativo. Desde entonces, con cada nuevo sexenio, se han emprendido reformas educativas a través de las cuales el presidente en turno, de acuerdo con su ideología, y siguiendo las corrientes políticopedagógicas imperantes, le da un nuevo enfoque a dicha práctica evaluativa.

Estas reformas educativas se presentan ante la sociedad como una solución para mejorar el servicio que se presta, las cuales implican un cambio de planes y programas de estudio en la educación básica. Un ejemplo de ello es la Ley General del Servicio Profesional Docente (LGSPD), derogada recientemente, la cual ostentaba que el punto medular de la problemática del sector educativo eran los profesores. Esto quedaba de manifiesto dentro del Plan Nacional de Desarrollo (PND) 2013-2018 (Gobierno de la República, 2013), el cual establecía que era necesario cambiar a un sistema de profesionalización de la carrera docente: el método para lograrlo era el fortalecimiento de los procesos de formación inicial y selección de los docentes. "La necesidad de contar con maestros, directores y supervisores mejor capacitados destaca como la principal vía para mejorar la calidad de la educación básica” (Gobierno de la República, 2013, p. 61). Por tal motivo era necesaria una evaluación del desempeño docente, lo que implicaba el someterse a una prueba estandarizada para el ingreso, permanencia o promoción.

Con el cambio de Gobierno federal, se retoma la discusión en torno al tema con la finalidad de llegar a un acuerdo nacional que recoja la visión de los diferentes actores de la educación. Así, se redacta el "DECRETO por el que se reforman, adicionan y derogan diversas disposiciones de los artículos 3o., 31 y 73 de la Constitución Política de los Estados Unidos Mexicanos, en materia educativa" (Diario Oficial de la Federación [DOF], 15 de mayo de 2019). Este caracteriza a los maestros y maestras de la siguiente manera:

Son agentes fundamentales del proceso educativo y, por tanto, se reconoce su contribución a la transformación social. Tendrán derecho de acceder a un sistema integral de formación, de capacitación y de actualización retroalimentado por evaluaciones diagnósticas, para cumplir los objetivos y propósitos del Sistema Educativo Nacional (DOF, 15 de mayo de 2019, párr. 8). 


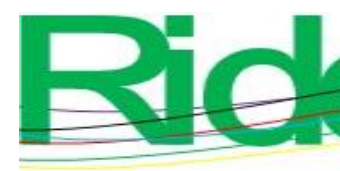

Revista Iberoamericana para la Investigación y el Desarrollo Educativo ISSN $2007-7467$

También se estipula que en las evaluaciones se considerarán los conocimientos, las aptitudes y la experiencia necesarios para guiar el proceso de aprendizaje.

A partir de lo anterior se propone la siguiente pregunta de investigación: ¿qué relación hay entre la evaluación del desempeño docente en su práctica y la evaluación docente a través de una prueba estandarizada?

La valoración del desempeño docente debe ser un proceso sistemático del cual se obtengan datos confiables que permitan comprobar el efecto educativo en los alumnos, donde se perciba el logro de aprendizajes y desarrollo de competencias; pero una evaluación estandarizada que homogeniza tanto a maestros como alumnos y que parte de situaciones hipotéticas no es el instrumento idóneo para evaluar todo lo que el profesor está realizando en su aula ante las diferentes circunstancias que se le presentan en su cotidianidad laboral.

Para Flores (2008), el desempeño docente:

Involucra un conjunto de decisiones, actividades y tareas, en las que participa el profesor en un contexto institucional, orientado a la formación de personas capaces de aportar y transformar su entorno socio-cultural; su evaluación es un requisito indispensable para mejorar la calidad educativa (p. 82).

La problemática ahora es establecer en la evaluación lo que se quiere medir y cómo hacerlo, por ejemplo, los instrumentos estandarizados que se han utilizado solo arrojan un puntaje para calificar a los profesores, lo cual ha servido para avanzar en los niveles de carrera magisterial, o el ser catalogado como idóneo o no para ingresar, desempeñar la función docente o lograr una promoción dentro del sistema educativo, pero no proporcionan información sobre las condiciones del trabajo docente en el día a día, con el fin de identificar sus fortalezas, así como las áreas de oportunidad, lo cual permite mejorar el proceso de enseñanza, y por ende, el del aprendizaje de los estudiantes.

En la tabla 1, siguiendo a Flores (2008), se pueden observar diferentes modelos de evaluación de desempeño docente. 


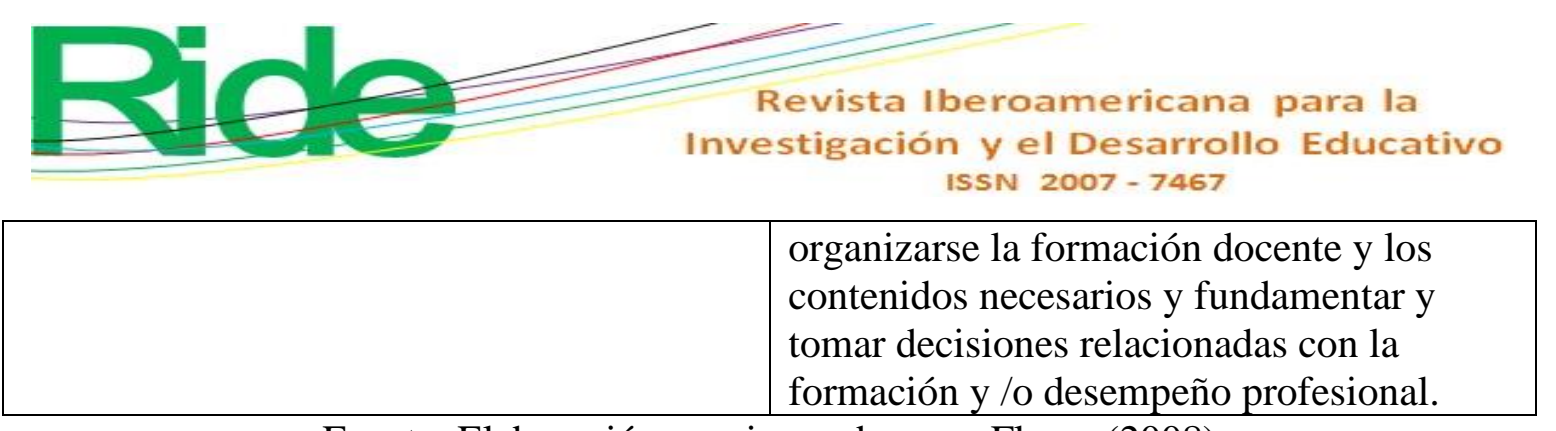

Fuente: Elaboración propia con base en Flores (2008)

De acuerdo con esta categorización, la evaluación docente en México consistía en aplicar una prueba estandarizada a los alumnos. Sin embargo, adelantándonos un poco, los docentes participantes en este estudio expresan que los ítems que se encontraban en el instrumento no eran acordes a las necesidades reales de los estudiantes ni respetaban la diversidad existente en cada aula; a pesar de ello, el resultado obtenido por el grupo tenía repercusiones para valorar el trabajo del docente; además, el profesorado presentaba pruebas de gran calado que homogenizaban al magisterio.

La evaluación centrada en estándares se propuso tanto en la evaluación universal como en los exámenes de ingreso, promoción y permanencia establecidos en la LGSPD, esto mediante un examen estandarizado aplicado a los docentes, de quienes se emitieron juicios sobre su desempeño sin considerar su actuación frente a las situaciones reales, solo según las ficticias que se mostraban en dicho instrumento. Para Santiago, McGregor, Nusche, Ravela y Toledo (2012):

Los exámenes estandarizados no consideran el contexto específico al que se enfrentan los maestros e ignoran los objetivos específicos de las escuelas donde trabajan. Además, la evaluación no es realizada por un evaluador que esté en mejor posición de entender las circunstancias específicas del maestro, por ejemplo, alguien de gestión escolar o supervisión, de manera que pueda ofrecer retroalimentación bien informada para que el docente responda mejor a las necesidades locales. En pocas palabras, los instrumentos que se utilizan actualmente carecen de interacción con el mundo real de la escuela-aula (p. 122).

La contraparte son los modelos centrados en la práctica reflexiva y en el comportamiento del docente en el aula. Estos proporcionan datos sobre lo que realmente realiza el profesorado en su trabajo con los estudiantes, lo cual brinda la posibilidad de detectar las deficiencias que se tienen, desde la planificación de clases que se hace con base en las características del grupo hasta su ejecución frente a este, lo que permite, a su vez, conocer sus debilidades y tomar decisiones para abatir las problemáticas vividas en el aula. 


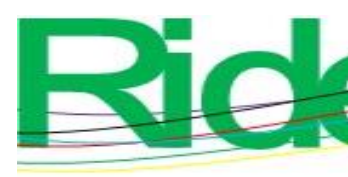

Revista Iberoamericana para la Investigación y el Desarrollo Educativo ISSN $2007-7467$

Santos Guerra (citado en Flores, 2008) concuerda en que la evaluación del desempeño docente debe ser una herramienta para transformar la práctica, con el fin de que cada profesor pueda estar mejorando su rol en el proceso de aprendizaje, y así, los alumnos logren la movilización de saberes de manera eficaz. Con todo ello se deja de lado, además, el etiquetar a los docentes en buenos o malos, idóneos o no idóneos, o en acreedores a estímulos económicos o no.

Por último, Sanmartí (2010) precisa que es dudoso que los profesores solo por estar evaluándose constantemente logren superar los problemas que tienen en su práctica docente; por el contrario, es necesario que se les apoye con los medios tanto para aprender como para enseñar y, por consecuencia, se impacte en el aprendizaje de los alumnos.

\section{Método}

Esta investigación se realizó por medio de un paradigma pragmático que considera que el conocimiento surge de las acciones, situaciones y condiciones que anteceden. Otorga mayor énfasis a lo que funciona, así como a la solución del problema, por tal motivo los investigadores utilizan diferentes enfoques (Creswell, 2003). Es de tipo mixto, de procedimiento secuencial exploratorio correlacional. La primera fase es de corte cualitativo con la finalidad de explorar el objeto de estudio de acuerdo con la perspectiva de los docentes, a través de la pregunta de investigación: ¿cómo evaluar el desempeño docente desde la perspectiva de los profesores? Lo anterior permitió elaborar un instrumento para valorar el desempeño docente.

La segunda fase consistió en la aplicación de dicho instrumento para valorar el desempeño docente a través de observaciones de jornadas de la práctica profesional de los profesores de la zona escolar 125. El instrumento está compuesto de 77 reactivos que integran las dimensiones Planificación de clases, Procedimientos metodológicos, Medios de enseñanza, Ambientes de aprendizaje y Práctica docente. Tiene una confiabilidad de 0.957, un puntaje de 0.865 en la validez de constructo. La técnica del jueceo por expertos para la validez de contenido arrojó, por su parte, un ICI (Índice de concordancia interobservador) de $96.10 \%$.

La aplicación del instrumento arrojó datos cuantitativos que se sometieron a pruebas estadísticas descriptivas e inferenciales, con el fin de rechazar o no las hipótesis planteadas. En este documento se exponen los resultados de la fase cuantitativa y el contraste de las dos 


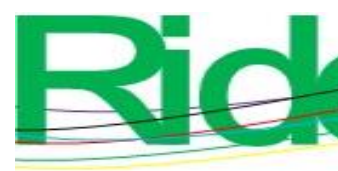

Revista Iberoamericana para la Investigación y el Desarrollo Educativo ISSN $2007-7467$

fases del estudio. Se tomó como guía la siguiente pregunta: ¿qué relación hay entre la evaluación del desempeño docente en su práctica, con la evaluación docente a través de una prueba estandarizada? El presente estudio se trabajó con una muestra 135 docentes que laboran en cinco zonas escolares que integran el sector educativo 125.

\section{Resultados}

De acuerdo con las fases de presente estudio, la aplicación del instrumento permitió recoger información sobre el desempeño docente en su práctica profesional dentro del aula. Su análisis arrojó los siguientes resultados cuantitativos, además de proporcionar un contraste entre las fases cualitativa y cuantitativa, lo cual se presenta a continuación.

\section{Desempeño docente en la práctica profesional}

Como se puede apreciar en la figura 1, al aplicar el instrumento para la evaluación del desempeño docente, solo en la dimensión Ambientes de aprendizaje los profesores obtuvieron un porcentaje mayor a $50 \%$; en las demás que integran este instrumento de evaluación no se alcanza ese porcentaje.

La primera dimensión es la denominada Planificación de clases. Allí se encontró que, en la muestra representativa, $53.84 \%$ presentó planificaciones de clases que no fueron elaboradas por los docentes participantes, ya que fueron copiadas, descargadas de Internet o compradas, por lo tanto, se infiere que no se realizaron partiendo de las necesidades, intereses, e incluso posibilidades de los alumnos atendidos.

Asimismo, se observó que $5.1 \%$ de las unidades de análisis plantea actividades diferenciadas para los niveles de desempeño de sus alumnos, es decir, las actividades que los docentes presentan a sus alumnos son planificadas para un grupo homogéneo, sin atender a los intereses, las necesidades, estilos y ritmos de aprendizaje que cada uno presenta, limitando en gran medida las adecuaciones necesarias. Por medio de estos resultados se puede apreciar que los docentes utilizan la planificación didáctica como un requisito administrativo, la cual deberán de presentar al director para rendir cuentas, pero no se está usando como una herramienta que guíe su práctica profesional, donde se planteen modos de actuar frente a situaciones que se pudieran dar al interior del aula de clase.

También se encontró que $51.28 \%$ de la muestra incluye diferentes instrumentos de evaluación. Entre los más frecuentes se encuentran las listas de cotejo o de control, que son utilizadas para registrar si los alumnos trabajaron, se portaron bien o cumplieron con tareas; 


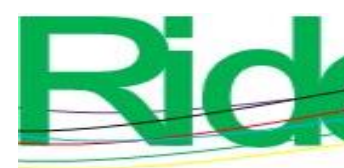

Revista Iberoamericana para la
Investigación y el Desarrollo Educativo
ISSN $2007-7467$

por lo que se le da menor importancia al proceso que los lleva al logro de los aprendizajes esperados, o a los propósitos que se marcan en los programas de cada grado. La responsabilidad de este proceso recae de forma directa en el profesor; solamente $2.5 \%$ plantea la coevaluación y $5.1 \%$ la autoevaluación.

Cabe destacar que una planificación bien diseñada, o el ponerla en marcha tal y como se planeó, no garantiza el éxito en la práctica o el logro de aprendizajes y el desarrollo de competencias, debido a que esta es una decisión previsoria de varios eventos que podrían pasar en la realidad, pero no hay certeza de que estos en efecto sucederán.

Por lo tanto, la planificación es una herramienta que guía el proceso educativo dentro del aula de clases, la cual puede ser modificada por las problemáticas que se presentan, pero se tienen que diseñar actividades de aprendizajes acordes, que atiendan a los alumnos con quienes se está trabajando, tomando en cuenta lo que se espera que se aprenda, además del cómo aprenden los alumnos. Realizar la planificación no solo es llenar los elementos de un formato de acuerdo con el programa de estudio vigente, sino es el momento en el que profesor articula sus conocimientos disciplinarios, pedagógicos, curriculares y su experiencia docente en torno a situaciones de aprendizaje.

En lo referente a la dimensión denominada Procedimientos metodológicos, se encontró que la muestra del estudio alcanzó la cifra de $43.5 \%$ como indicador de logro. Se constató que $61.53 \%$ utiliza procedimientos que orientan al alumno para que desarrolle sus competencias de forma independiente, sin embargo, no se garantiza que dichas competencias realmente se desarrollen.

También arrojó que $56.41 \%$ de los participantes en esta investigación, en su desempeño docente, pone en práctica actividades para propiciar en el alumno el desarrollo de su pensamiento reflexivo, pero las videograbaciones muestran que solo $35 \%$ de los docentes realiza funciones de guía, orientador: la dirección del proceso de aprendizaje sin anticiparse a los razonamientos y juicios de los niños, con lo que se propicia que el alumno sea el centro de aprendizaje.

Los procedimientos metodológicos incluyen las actividades propias del docente como la planificación, organización y orientación de las actividades del alumno en el proceso de aprendizaje, pero sin descuidar el interés y los estilos de aprendizajes de sus estudiantes. De tal forma que toma relevancia el enfoque didáctico que el profesor aplica, además del que se 


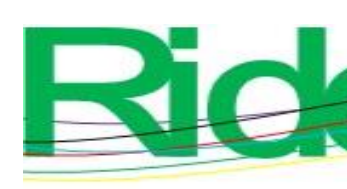

Revista Iberoamericana para la Investigación y el Desarrollo Educativo ISSN $2007-7467$

establece a través de las propuestas en los programas para el logro de aprendizajes y el desarrollo de competencias.

De los datos se encuentra que $25 \%$ de los participantes estimula la búsqueda de información en diversas fuentes, lo cual le permite al alumno analizar diversos textos con el fin de tomar una decisión informada, y así desarrollarse en esta era de la información. Además, $43.58 \%$ de los profesores aplica un enfoque didáctico que permite a los discentes el desarrollo de su independencia cognitiva, pero solamente $7.6 \%$ utiliza diferentes niveles de ayuda para que el alumnado pueda reflexionar sobre su error o problemática a la que se enfrenta con el fin de rectificarlo o superarlo.

El término metodología no solo implica el enfoque del profesor o el rol de alumno en el proceso de aprendizaje, sino que también considera a otro elemento en juego: los recursos con que cuenta el docente para desarrollar su labor, denominados en este estudio como medios de enseñanza. Es decir, de acuerdo con Latorre y Seco (2013), la metodología docente debe contemplar el enfoque del profesor, el rol del alumno y los medios de enseñanza; enfatizar solo un elemento no implicaría hablar de metodología.

La constante evolución tecnológica obliga al profesor a que no se mantenga en el uso exclusivo de los recursos que le fueron eficaces hace tiempo, ya que los alumnos de hoy son niños que están creciendo en la era de la tecnología. Por este motivo, dentro del repertorio de recursos docentes, son necesarias las tecnologías de la información y la comunicación (TIC). Sin embargo, en contraste, en la dimensión Medios de enseñanza los profesores alcanzaron solo $17.52 \%$ del porcentaje de logro. Este resultado denota que la mayor parte de la muestra representativa de esta investigación no utiliza recursos didácticos que estén más allá del cuaderno y el libro de texto, lo que ocasiona desinterés por parte de los alumnos hacia las actividades planteadas. Si este panorama se suscita de forma muy parecida en otras zonas escolares, tanto de la región como del estado o del país, puede explicarse el por qué a los alumnos les resulta más atractivo el tiempo en casa: cuentan con acceso a diversas tecnologías.

Ahora bien, $23.07 \%$ de los participantes utilizan actividades lúdicas durante su práctica docente con el fin de motivar a los alumnos en su proceso de aprendizaje, $10.25 \%$ planifica actividades con material concreto, $2.5 \%$ apoya sus clases con material visual y $38.46 \%$ utiliza material escrito como un recurso complementario. Entre los medios más recurrentes por parte del profesor, 100 \% utiliza el pizarrón, además del discurso oral. 


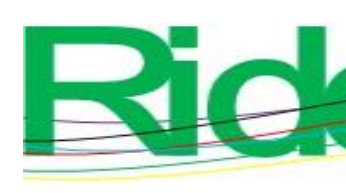

Revista Iberoamericana para la Investigación y el Desarrollo Educativo ISSN $2007-7467$

En la dimensión Ambientes de aprendizaje los docentes obtienen su mejor porcentaje de logro: $67.39 \%$. Esta cifra permite suponer que los profesores, en su práctica profesional, consideran los aspectos físicos del aula, los contenidos a trabajar, los materiales en que se pueden apoyar y a los alumnos. Asimismo, $82.05 \%$ de la muestra de este estudio, en su espacio y tiempo de trabajo, con sus actitudes hacia los alumnos, favorece un clima para que estos puedan expresar sus sentimientos, así como el respeto en las relaciones que establecen con sus pares. Aunado a ello, $66.66 \%$ de los docentes propicia un ambiente donde los niños desarrollan sus competencias comunicativas al permitir realizar diferentes argumentaciones.

En esa misma línea, $89.74 \%$ de los participantes utilizan un lenguaje adecuado para los niños y también demuestran respeto a los estudiantes y a sus trabajos. Con estas actitudes se promueven la formación de hábitos y valores por medio del ejemplo. Pero $51.29 \%$ de los participantes no favorece la inclusión educativa ni atiende a la diversidad, debido a que no se desarrollan actividades para los diferentes estilos de aprendizaje o para las necesidades específicas de los estudiantes, es decir, no aplica adecuaciones.

Para la Oficina Regional de Educación de la Unesco para América Latina y el Caribe [Orealc] (2008), la escuela es la encargada de hacer la diferencia en el rendimiento de los alumnos, debido a que las variables asociadas a la institución propician una disminución en las desigualdades de aprendizaje. Además, según la misma Orealc (2008), un buen ambiente de aprendizaje es la variable más influyente en el rendimiento escolar, "por lo tanto, la generación de un ambiente de respeto, acogedor y positivo es esencial para promover el aprendizaje entre los estudiantes" (p. 178).

En esta dimensión se puede presenciar la relación entre el currículo formal, el oculto y el real. El primero se entiende como el programa vigente para la educación primaria elaborado por la Secretaría de Educación Pública (SEP) en el 2011 y el cual le da prioridad al logro de aprendizajes y al desarrollo de competencias; mientras que el real o vivido corresponde a cómo el profesor ejecuta lo establecido por la SEP en su práctica docente, claro que para ello es indispensable la interpretación que él hace sobre el formal, de acuerdo con su formación e ideología.

La última de las dimensiones, la dimensión de la Práctica docente arrojó 39.79 \% de logro. Aquí se desprenden los siguientes resultados: $48.71 \%$ de los docentes lleva a la práctica la planificación presentada con autoridad inmediata, ejecuta la mayoría de las actividades planteadas. Además, $51.28 \%$ aplicó situaciones didácticas que conllevan al logro 
Figura 1. Porcentaje de logro del desempeño docente

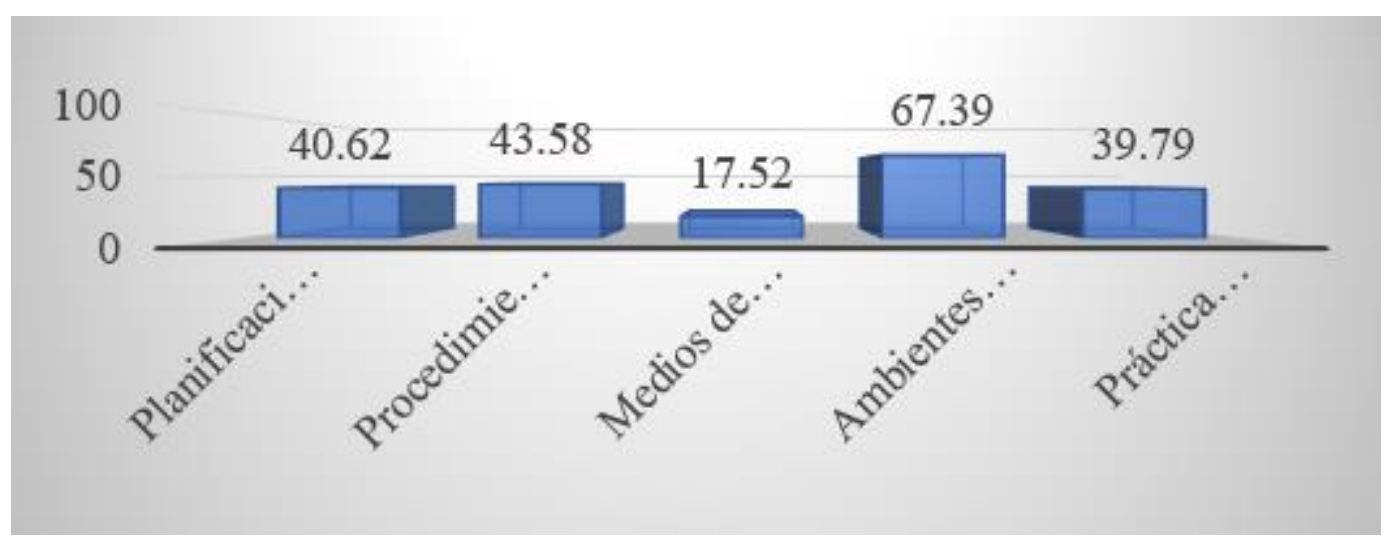

Fuente: Elaboración propia

\section{Relación entre estudios del docente y una evaluación estandarizada}

Para los datos sobre el grado de estudios de los docentes y los resultados obtenidos en una evaluación estandarizada, se aplicó la prueba de la ji al cuadrado para corroborar la existencia de asociación entre ellos. Los resultados obtenidos se aprecian en la tabla 2. De acuerdo con la regla de decisión planteada, el valor que arrojó el estadístico es de 96.075 al ser este menor al valor crítico de 101.879 , y con una significancia de 0.311 mayor al 0.05 establecido, por lo cual no se rechaza la $\mathrm{H}_{0}$ : no existe asociación entre el grado de estudios y el puntaje obtenido en las pruebas estandarizadas.

Tabla 2. Asociación entre el grado de estudios del profesor y el rendimiento en una prueba estandarizada

\begin{tabular}{|c|c|c|c|}
\hline \multicolumn{4}{|c|}{ Pruebas de chi-cuadrado } \\
\hline & Valor & Gl & $\begin{array}{c}\text { Sig. asintótica } \\
\text { (bilateral) }\end{array}$ \\
\hline $\begin{array}{c}\text { Chi-cuadrado de } \\
\text { Pearson }\end{array}$ & $96.075^{\text {a }}$ & 90 & .311 \\
\hline $\begin{array}{c}\text { Razón de } \\
\text { verosimilitudes }\end{array}$ & 65.862 & 90 & .974 \\
\hline
\end{tabular}

Fuente: Elaboración propia 


\section{Correlación entre desempeño docente en la práctica profesional y el desempeño docente evaluado a través de una prueba estandarizada}

Como se puede apreciar en la tabla 3, al realizar el análisis estadístico se encontró que existe una mínima correlación entre el puntaje del profesor en una evaluación estandarizada y su desempeño docente observado en este estudio, pero como el nivel de significa es mayor a 0.05 , no se cuenta con los datos necesarios para realizar afirmaciones sobre esta relación, por tal motivo no se rechaza la $\mathrm{H}_{0}$ : no existe relación entre el desempeño del profesor con el rendimiento del profesor en una evaluación estandarizada.

La valoración del desempeño docente es un proceso sistemático para obtener datos confiables que permitan comprobar el efecto educativo en los alumnos, donde se perciba el logro de aprendizajes y el desarrollo de competencias, por ende, una evaluación estandarizada que homogeniza tanto a los maestros no es el instrumento idóneo para evaluar todo lo que realmente están realizando en el salón de clases, donde se suscitan diferentes condiciones al momento de estar en plena labor de enseñanza.

La evaluación del desempeño docente debe ser un proceso continuo que ofrezca un juicio sobre la intervención del profesor en el proceso de aprendizaje del alumno; una en la que se consideren los diferentes factores que intervienen en su práctica profesional con la finalidad de detectar las áreas de oportunidad; por ende, debe ser una herramienta para transformar la práctica con repercusión directa en el aprendizaje de los alumnos y, con ello, dejar de ser una etiquetadora maniqueísta de docentes: buenos o malos, idóneos o no idóneos, o aspirantes o no a estímulos económicos.

Aun con todo, el someter a los profesores a una evaluación por medio de pruebas de gran calado no garantiza que se superen los problemas presentes en su práctica docente, sino que es necesario que se les apoye con los medios tanto para aprender como para enseñar, y de tal forma se impacte en el aprendizaje de los alumnos. Así pues, la evaluación del desempeño docente debe estar centrada en la práctica profesional, lo cual otorga evidencia sobre la eficacia de su desenvolvimiento educativo, y devela áreas de oportunidad sobre las cuales hacer una reflexión en conjunto, maestro y evaluador, con el fin de resarcir las problemáticas detectadas. El papel de la evaluación del desempeño docente debe ser el de un

aliado para apoyar al profesor a tener una mejora continua en su profesión y no el de ser un medio para amenazar la continuidad de este. 


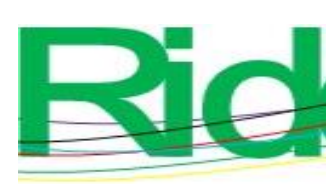

Revista Iberoamericana para la Investigación y el Desarrollo Educativo ISSN $2007-7467$

Tabla 3. Correlación entre desempeño docente en la práctica profesional y el desempeño docente evaluado a través de una prueba estandarizada

\begin{tabular}{|l|l|c|}
\hline \multicolumn{2}{|c|}{ Correlaciones } \\
\hline \multicolumn{2}{|c|}{} & Desempeño docente \\
\hline \multirow{2}{*}{$\begin{array}{l}\text { Puntaje de profesor en } \\
\text { evaluación estandarizada }\end{array}$} & Correlación de Pearson & 0.099 \\
\cline { 2 - 3 } & Sig. (bilateral) & 0.686 \\
\hline
\end{tabular}

Fuente: Elaboración propia

\section{Relación desempeño docente con los años de trabajo en la institución escolar}

Del análisis estadístico de estas variables por medio del coeficiente de correlación de Pearson, se obtuvo un nivel de significancia menor que 0.05 , por tal motivo se rechaza la hipótesis $\mathrm{H}_{0}$ : no existe relación entre el desempeño del profesor con los años que el docente tiene trabajando en la institución. Como se puede apreciar en la tabla 4, existe una correlación de -0.416, la cual, de acuerdo con Hernández, Fernández y Baptista (2006), es considerada como media entre las variables; no obstante, como se puede apreciar, es una correlación negativa, lo que implica que a mayor cantidad de años de estar trabajando en la institución el nivel de desempeño docente es menor, mientras que a menor cantidad de años de permanecer laborando en la misma institución su desempeño docente es mejor.

La peculiaridad de los docentes que tienen cuatro o menos años de laborar en la institución educativa es la motivación que se observa. Ellos realizan su trabajo con gran alegría, así como con una energía que los impulsa a dar el extra en su labor, se esfuerzan para que los alumnos tengan un buen proceso de aprendizaje. Y contrario a esto, se percata que los profesores que tienen más años trabajando en la misma primaria son apáticos o reacios a innovar en su práctica docente, se percibe que se encuentran en una zona de confort que les impide analizar críticamente su desempeño. El factor determinante es la presencia o ausencia de motivación en los docentes. La motivación, según Maslow (citado en Pila, 2012), se define de la siguiente forma: 

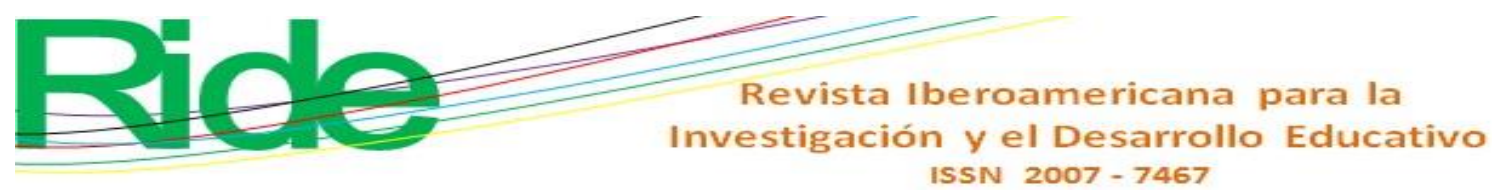

Es el lazo que lleva esa acción a satisfacer la necesidad. En este sentido, la motivación se convierte en un activador de la conducta humana. Los estados motivacionales, lo mismo que los actitudinales, se generan por efecto de un conjunto de factores o variables que se interaccionan (p. 36).

Teniendo en cuenta lo anterior, la motivación conduce el comportamiento del docente para satisfacer sus necesidades. Los profesores que tienen poco tiempo de laborar en una institución educativa tratan de desarrollar su práctica profesional de la mejor manera, ya que en ellos existe la necesidad o el deseo de ser reconocidos como buenos maestros por parte de sus alumnos, por parte de los padres de familia, por parte de los directivos.

McClelland (citado en Pila, 2012) expresa que existe en los docentes una insatisfacción por la necesidad de realización, es decir, los profesores tienen el interés de desarrollarse como profesionistas exitosos, por lo tanto, sus acciones son con el fin de sobresalir o destacar con buenos resultados. Esto le permite aceptar diferentes responsabilidades o retos para demostrar su desempeño.

Tabla 4. Relación desempeño docente con los años de trabajo en la institución escolar

\begin{tabular}{|l|l|c|}
\hline \multicolumn{2}{|c|}{ Correlaciones } \\
\hline \multicolumn{2}{|c|}{} & Antigüedad en la escuela \\
\hline \multirow{2}{*}{ Desempeño docente } & Correlación de Pearson & - \\
\cline { 2 - 3 } & Sig. (bilateral) & $0.416^{* *}$ \\
\hline
\end{tabular}

Fuente: Elaboración propia

\section{Relación años de servicio y desempeño docente}

Para el análisis de las variables que intervienen en esta hipótesis, se realizó el coeficiente de Pearson, el cual arrojó una significancia menor a 0.05 , por lo cual no se acepta la hipótesis $\mathrm{H}_{0}$ : no existe relación entre la experiencia laboral del docente con su desempeño docente. En la tabla 5 se muestra un puntaje de correlación entre las variables de 0-.316, considerada como de grado media pero, una vez más, negativa, lo cual implica que al tener más años de servicio frente a grupo su desempeño docente en el aula decrece. En esta investigación lo anterior se manifiesta en los profesores de nuevo ingreso o considerados noveles, quienes, a pesar de algunas deficiencias en el dominio de enfoques didácticos 


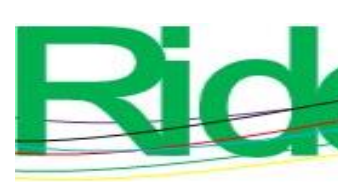

Revista Iberoamericana para la Investigación y el Desarrollo Educativo ISSN $2007-7467$

aplicado constantemente, comienza a sentirse renuente a los cambios, presenta un desinterés en el quehacer docente, y entra en una etapa de negatividad o conformismo.

Tabla 5. Relación años de servicio con su desempeño docente

\begin{tabular}{|l|l|c|}
\hline \multicolumn{2}{|c|}{ Correlaciones } \\
\hline \multicolumn{2}{|c|}{} & Desempeño docente \\
\hline \multirow{2}{*}{ Años de servicio docente } & Correlación de Pearson & - \\
\cline { 2 - 3 } & Sig. (bilateral) & $0.316^{* *}$ \\
\hline
\end{tabular}

Fuente: Elaboración propia

\section{EI desempeño docente desde la práctica diaria}

De la figura 2 se deduce que un profesor con buen desempeño dentro del salón de clases no es aquel que logra entrenar a sus estudiantes para resolver adecuadamente un examen que proporciona, además, una puntuación para la escuela o para su propio expediente, sino aquel que realmente se preocupa por la formación integral de estos, que está al pendiente de su condición, así como de lo necesario para que puedan enfrentar los problemas de su realidad inmediata.

Este tipo de docentes debe conocer a los niños que están a su cargo, así como a la comunidad, la situación socioeconómica de donde procede cada uno de ellos y del mismo lugar en que se encuentra ubicada la institución, ya que esto le permite realizar una planificación de actividades contextualizada, es decir, acorde a las necesidades e intereses de sus estudiantes, donde él pueda diseñar una diversidad de actividades que atienda a los diferentes estilos de aprendizaje.

Con la característica anterior de la planificación, las estrategias didácticas que plantee serán atractivas para los estudiantes debido a que presentan situaciones de la realidad que ellos viven día a día, por lo tanto, encontrarán un sentido práctico o útil al aprendizaje que están construyendo, así como a las competencias que está desarrollando.

El docente, al estar creando las actividades necesarias para su grupo, tiene que recordar constantemente que estas girarán en torno al niño y su proceso de aprendizaje, además de los recursos con que cuenta realmente para hacer un uso eficiente de ellos. En ocasiones esto pasa desapercibido por los profesores. Cuando copian una planificación se 


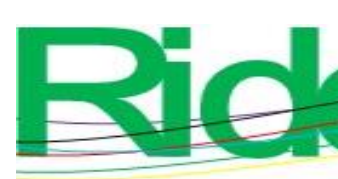

Revista Iberoamericana para la
Investigación y el Desarrollo Educativo
ISSN $2007-7467$

mencionan estrategias de videos o actividades de Internet, pero en la escuela o en el aula no se cuenta con lo necesario.

Por ello es indispensable que desde este primer bosquejo de las actividades que se pretenden desarrollar el maestro considere los recursos que utilizará y que están a su verdadero alcance. Si es un video, un ejercicio impreso, o algún material concreto, deberá de adquirirlo previamente para poder ejecutar su planificación, porque después se cae en una improvisación dentro del aula.

Esta organización realizada puede ser flexible y adecuarse a ciertas circunstancias que se presentan al interior del aula, lo cual no significa que hay luz verde para cambiar constantemente las actividades en su totalidad. Al respecto, conviene recordar que la planificación es una "propuesta de trabajo dinámica que podrá modificarse o replantearse cuando sea necesario, tomando como referencia las características de los alumnos, la viabilidad de lo que se propone, los factores que favorecen u obstaculizan el aprendizaje de los niños" (SEP, 2013b, p. 16).

De esto se desprende la sistematización de las actividades, los recursos, así como el tiempo con el que dispone el profesor, ya que estos elementos en su conjunto son la propuesta de trabajo. Mediante su ejecución, los alumnos lograrán construir su aprendizaje, porque en su diseño se contemplaron las acciones más viables para lograrlo. También se deben incluir los instrumentos de evaluación que proporcionen información tanto de las actividades del alumno como del docente.

Los profesores que alcanzaron un nivel destacado en su evaluación del desempeño docente dentro de esta investigación plantearon dentro de su planificación y se observó en su práctica profesional el uso de materiales y recursos didácticos que favorecen el proceso de aprendizaje. No obstante, para que estos puedan tener un efecto positivo en el aprendizaje es primordial conocer con quiénes se está trabajando. El profesor debe identificar los estilos de aprendizaje, las necesidades e intereses de sus alumnos para elaborar los materiales apropiados. Existen maestros que recurren en la monotonía de recursos: solo manejan el discurso oral o el uso excesivo de ejercicios impresos, lo que ocasiona que los estudiantes no presten atención a la actividad o que su interés se pierda rápidamente. 

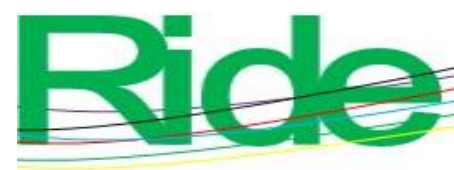

Revista Iberoamericana para la Investigación y el Desarrollo Educativo ISSN 2007 - 7467

Pero las evidencias de las evaluaciones les ayudan a reflexionar sobre su práctica profesional, ya que, al detectar algunas dificultades en los contenidos, reconocen las áreas de oportunidad de sus alumnos. Además, les permiten reorientar su práctica a través de los ajustes que establecen en su planificación, por lo tanto, diseñarán estrategias que impliquen un reto para propiciar la movilización de saberes de los estudiantes.

Un elemento que se repetía en los buenos profesores era la motivación por su quehacer diario. Quien se sabe valorado en su entorno laboral, tiene una buena relación con sus compañeros y con sus jefes inmediatos, siente satisfacción por las actividades que realiza, por ende, presenta gran interés en su trabajo, tiene objetivos claros y despliega todo su potencial para alcanzarlos. De acuerdo con la teoría motivacional de Maslow (citado por Pila, 2012), es necesario tener satisfechas las necesidades fisiológicas y de seguridad para acceder y cumplir con las sociales, que se pueden observar en la aceptación de los docentes en el centro de trabajo, así como en las relaciones que se establecen entre ellos, para la búsqueda del reconocimiento; motivar al profesor en su trabajo para alcanzar una valoración por parte de las personas dentro y fuera de la institución.

Figura 2. Buen desempeño docente

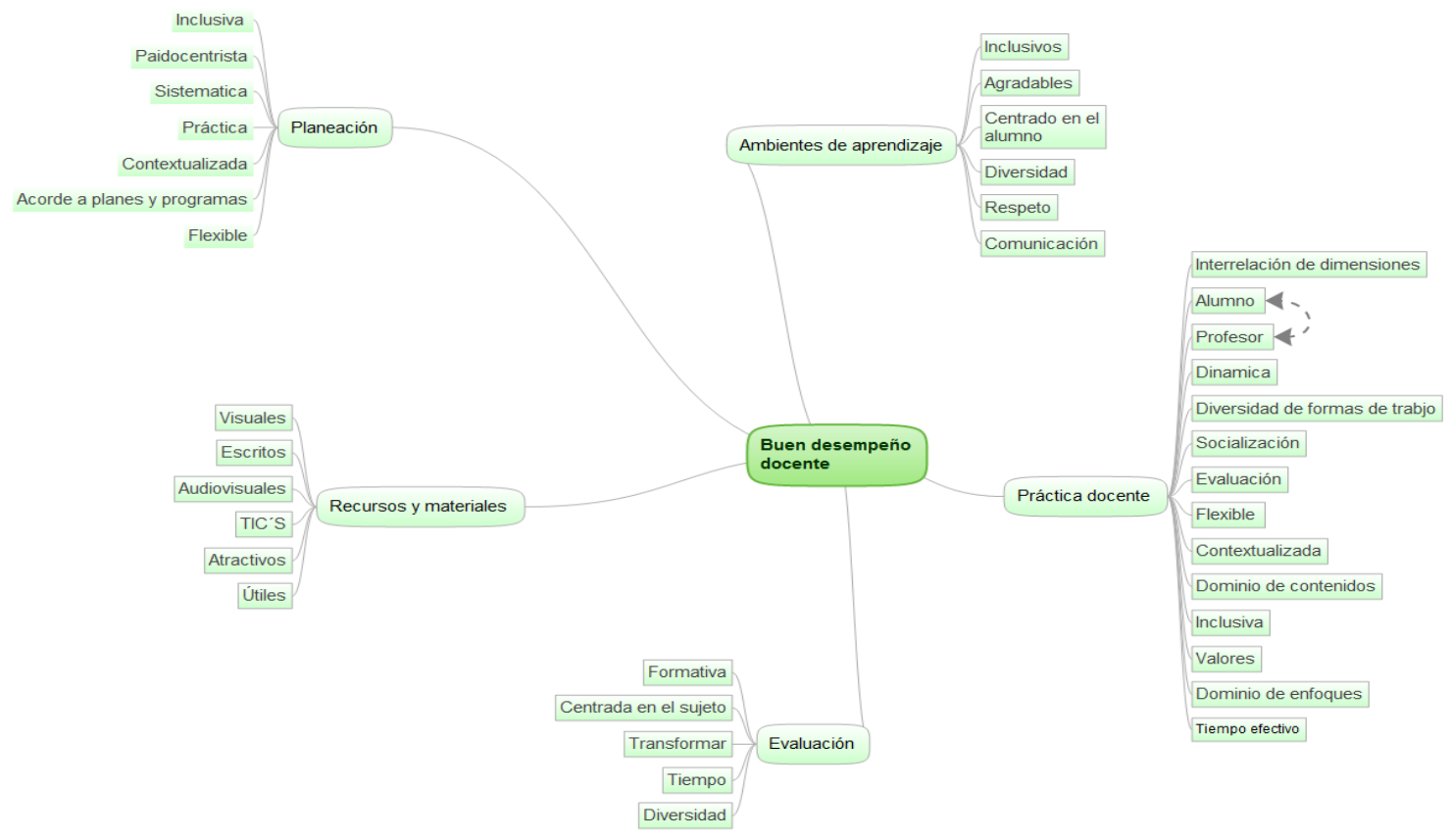

Fuente: Elaboración propia 


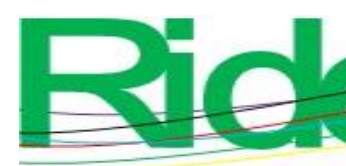

.

\section{Características del profesor con una buena práctica profesional}

Mediante el contraste de la información de ambas etapas de esta investigación fue posible acceder a las características de los profesores que obtuvieron un muy buen desempeño docente o excelente, las cuales quedaron plasmadas en la figura 3, integrada por varias subcategorías, una de ellas relacionada con el ser profesional, en la que se percibe a un sujeto que cuenta con el perfil docente que le permite desempeñar el cargo que ostenta.

Vale la pena destacar que los docentes de los que se deducen estas aptitudes o características no se conforman con poseer el título de licenciado en Educación Primaria, sino que buscan estar al día, esto es, se actualizan constantemente para enfrentar los retos que se le presenten en su práctica profesional.

Además, hacen un uso eficiente del tiempo que disponen para trabajar con los alumnos. En su práctica no existen los tiempos muertos, los niños permanecen activos durante toda la jornada escolar. También manejan de forma racional los recursos con que cuenta la institución para beneficio del alumnado. Este tipo de maestros no usan como excusa lo que les falta, sino que innovan, realizan adecuaciones para transformar las desventajas del contexto escolar en ventajas. Es decir, conocen las limitaciones que existen en su centro de trabajo, pero esto no los limita para realizar su quehacer docente de la mejor manera.

Otra subcategoría es la denominada Sin distractores. Por distractor se entiende cualquier situación u objetos que proporcionan un estímulo negativo a las personas, y ocasiona la pérdida de atención o desinterés por la actividad que se realiza.

A través de este estudio se ha hecho hincapié en que los docentes involucren las TIC en su práctica profesional con el propósito de diversificar los recursos didácticos y motivar a los alumnos, pero la tecnología solo ha llegado mediante el teléfono celular, lo cual ha afectado su desempeño, ya que los maestros lo usan constantemente para estar en contacto con las demás personas por medio de las redes sociales, permanecen gran tiempo conectados en ellas, con repercusiones en el trabajo áulico y en la atención de sus alumnos.

Un distractor más son las salidas del grupo de clases. En los profesores con un desempeño regular o malo se puede apreciar que ellos no permanecen la jornada de clases completa en aula realizando actividades de aprendizaje, debido a que se ausentan para platicar con los compañeros de trabajo, por ayudar a los directivos con el trabajo administrativo, o porque no se tienen los materiales plasmados en la planificación escolar y salen a buscarlos. 


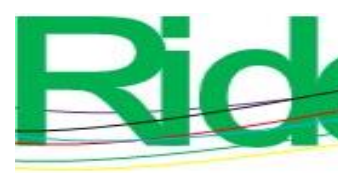

Revista Iberoamericana para la
Investigación y el Desarrollo Educativo
ISSN $2007-7467$

Al salir del aula incumplen con algunos de los rasgos de la normalidad mínima, como uso efectivo del tiempo en actividades de aprendizaje, o el iniciar a tiempo la jornada de clases, por ende, los alumnos permanecen solos. El rol del docente deber ser de guía en el proceso de aprendizaje, pero los estudiantes, al no contar con su presencia, coartan su aprendizaje, de ahí que los resultados que se obtienen no sean los idóneos.

Una característica en los profesores con buen desempeño docente es la ausencia de estos distractores dentro de su práctica profesional: permanecen la jornada escolar en el aula, destinan el tiempo para atender a las necesidades de los alumnos y a los procesos de aprendizaje.

Otra peculiaridad es la motivación que se observa en los profesores que tienen cuatro o menos años de laborar en la institución educativa. Ellos realizan su trabajo con gran alegría, así como con una energía que los impulsa a dar el extra en su labor, se esfuerzan para que los alumnos tengan un buen proceso de aprendizaje.

$\mathrm{Su}$ profesión es proporcionar un servicio a los demás, por lo tanto, en sus actos predomina el respeto por los alumnos y hacia su trabajo. En cada momento intentan dar el extra para que los estudiantes logren los aprendizajes esperados, aunque existan adversidades a su alrededor.

Estos docentes, además, no se conforman con solo alcanzar lo que establece el programa de estudio, sino que orientan sus esfuerzos para que el alumno pueda tener una formación integral como individuo que le permita enfrentar con éxito los retos que la sociedad le demande. Por lo tanto, no solo cuentan con el perfil requerido para ejercer la profesión, sino que tienen la vocación que le permite hacer su trabajo con gusto y encontrar una satisfacción en ello.

Una característica más de un buen docente es la de ser líder, no como alguien que ejerce su autoridad sobre los demás, solo por el cargo que ostenta, sino como alguien que se involucra en las relaciones personales que se dan en el aula, quien, a través de su comportamiento, influye en las conductas de sus estudiantes, gracias a lo cual los involucra en el trabajo colaborativo e individual para abatir las necesidades o debilidades detectadas en el plan anual y así alcanzar las metas establecidas. El líder, según Pascual (citado en Vázquez, 2012), "tiene una habilidad social para invadir el yo del seguidor y cambiar su voluntad hacia donde él quiere, logrando su confianza" (p. 17). 

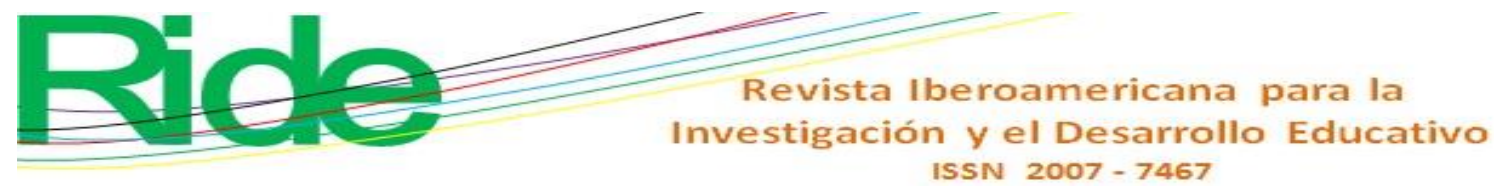

En palabras de Bass (citado en Vázquez, 2012), se trata de un líder transformador, que, a través del carisma, de la confianza brindada, así como de la motivación, inmiscuye a los alumnos en un proceso de respuestas positivas en su aprendizaje, habilidades y actitudes, aun cuando existan resistencias en el grupo.

Figura 2. Características del profesor con una buena práctica profesional

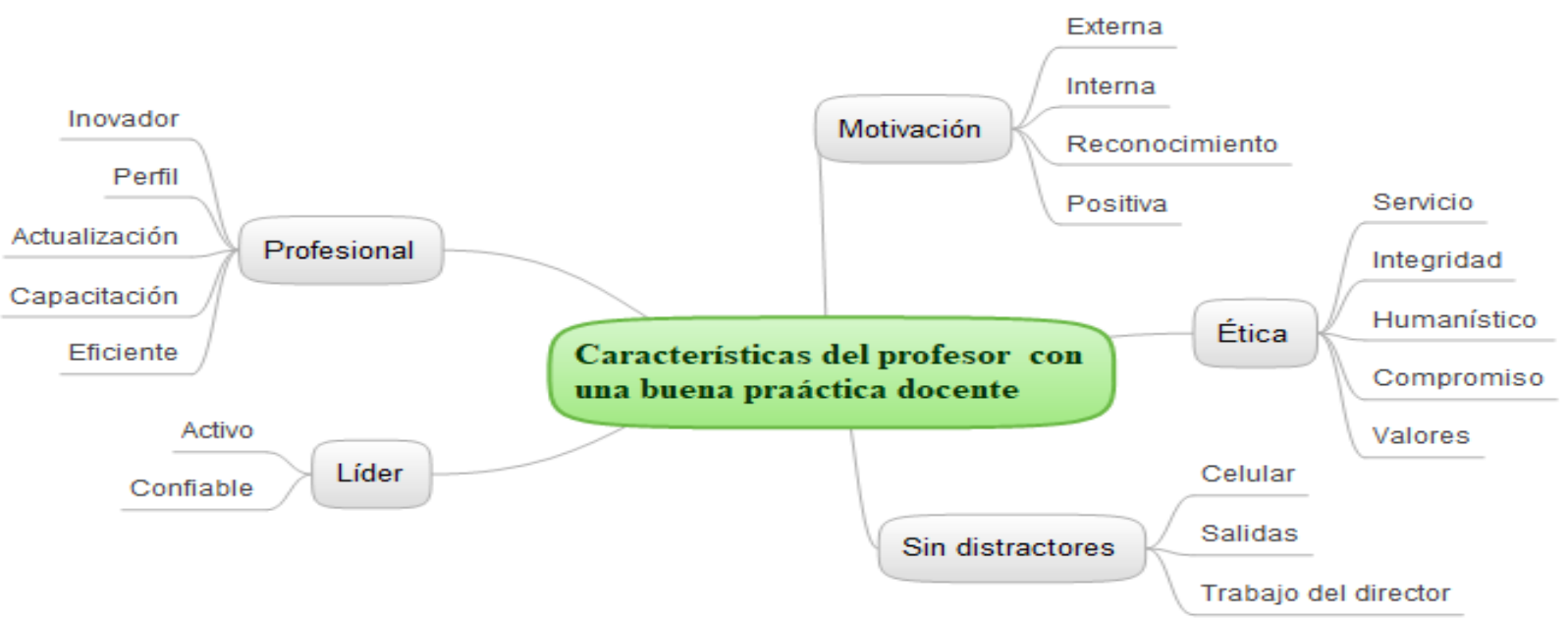

Fuente: Elaboración propia

\section{Discusión}

Las evaluaciones docentes han estado en una constante evolución. En un inicio, se implementaron las evaluaciones voluntarias, que pretendían la modernización y actualización del profesorado; además, a través de cursos y exámenes, podían acceder a una mejor remuneración económica. Sin embargo, en los últimos años se presenció un cambio drástico: este tipo de ejercicios se convirtió en obligatorio: el estrés y la incertidumbre comenzaron a imperar.

A lo largo del tiempo, una variable inmutable para emitir un juicio del desempeño docente han sido las pruebas estandarizadas descontextualizadas, las cuales, a través de un discurso de conocer las áreas de oportunidad del profesorado, solo han servido como una medida de comparación y descalificación de los docentes. Teniendo en cuenta esto, en esta investigación se concuerda con otras respecto a la necesidad de establecer diferentes instrumentos de evaluación (Aboites, 2012; Flores, 2008; Martínez, 2016; Sanmartín, 2010). Para Santiago et al. (2012), "los exámenes estandarizados no consideran el contexto 


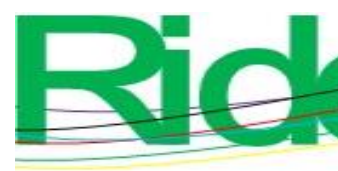

Revista Iberoamericana para la
Investigación y el Desarrollo Educativo
ISSN $2007-7467$

específico al que se enfrentan los maestros e ignoran los objetivos específicos de las escuelas donde trabajan" (p. 122).

De acuerdo con los resultados que se especifican en la tabla 3, que implicó no rechazar la $\mathrm{H}_{0}$ : no existe relación entre el desempeño del profesor con el rendimiento del profesor en una evaluación estandarizada, aquí se considera primordial el evaluar el desempeño docente desde la práctica profesional del profesor, ya que en ella realmente se tendrá información contextualizada del actuar de este, teniendo en cuenta lo que enfrenta día a día.

Por lo tanto, es indispensable prestar atención a diferentes dimensiones que integran el desempeño docente. En la presente investigación se encontró que $53.84 \%$ de la muestra presentó planificaciones de clases que no fueron elaborados por los docentes que participaron en esta investigación. Esto no es cualquier cosa si se toma en cuenta que, según Gómez (2008), la planificación es el esquema en donde el docente organiza su actuar atendiendo a su conocimiento práctico, el cual emerge y evoluciona en las diferentes situaciones que enfrenta. Mientras que para Monroy (1998) en esta los profesores plasman su conocimiento disciplinar, pedagógico y su ética profesional.

Por último, son igualmente importante los resultados estipulados en las tablas 4 y 5 , los cuales aportan evidencia para rechazar las hipótesis $\mathrm{H}_{0}$ : no existe relación entre el desempeño del profesor con los años que el docente tiene trabajando en la institución, así como $\mathrm{H}_{0}$ : no existe relación entre la experiencia laboral del docente con su desempeño docente. Pero la relación existente es negativa, por lo tanto, es necesario revisar las diferentes clasificaciones de las etapas del ciclo del servicio docente (Huberman, 1990; Serrano, 2012; Sikes, Measor y Woods, 1985) para retomar la experiencia docente como un ámbito para avanzar tanto en el organigrama vertical como horizontal. 


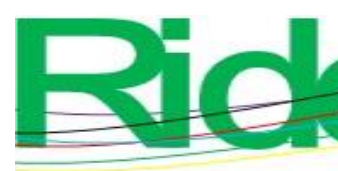

Revista Iberoamericana para la Investigación y el Desarrollo Educativo ISSN 2007-7467

\section{Referencias}

Aboites, H. (2012). La medida de una nación. Los primeros años de la evaluación en México: historia de poder y resistencia (1982-2012). México: Universidad Autónoma Metropolitana.

Creswell, J. W. (2003). Research: Qualitative, Quantitative and Mixed methods approaches. California. E.U.A: Sage. Segunda edición.

Diario Oficial de la Federación. (2013). La Ley General del Servicio Profesional Docente. México, D. F.

Diario Oficial de la Federación [DOF]. (15 de mayo de 2019). Decreto por el que se reforman, adicionan, y derogan diversas disposiciones de los artículos 3, 31, y 73 de la Constitución de los Estados Unidos Mexicanos, en materia educativa. Diario Oficial de la Federación.

Fierro, C., Rosas, L. y Fortoul, B. (1999). Transformando la práctica docente (1. ${ }^{\mathrm{a}}$ ed.). México: Paidós.

Flores, F. (2008). Las competencias que los profesores de educación básica movilizan en su desempeño profesional docente. (tesis de doctorado). Universidad Complutense de Madrid, España.

Gobierno de la República. (2013). Plan Nacional de Desarrollo 2013-2018. Ciudad d de México, México: Gobierno de la República.

Gómez López, L. (2008). Los determinantes de la práctica educativa. Universidades.

Hernández, R., Fernández, C. y Baptista Lucio, P. (2006). Metodología de la investigación (4. ${ }^{\mathrm{a}}$ ed.). México: McGraw-Hill.

Huberman, M. (1990). Las fases de la profesión docente. Ensayo de descripción y previsión. Qurriculum: Revista de Teoría, Investigación y Práctica Educativa, (2), 139-159.

Latorre Ariño, M., y Seco del Pozo, C. (2013). Metodología, estrategias y técnicas metodológicas. Lima. Perú.

Leinhardt, G. and Greeno, J. G. (1986). The cognitive skill of teaching. Journal of Educational Psychology, 78(2), 75-95.

Martínez, F. (2016). La evaluación de docentes de educación básica. Una revisión de la experiencia internacional. México: Fondo Editorial INEE. 


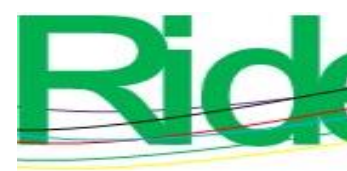

Revista Iberoamericana para la
Investigación y el Desarrollo Educativo
ISSN $2007-7467$

Monroy, M. (1998). El pensamiento didáctico del profesor: un estudio con profesores de ciencias histórico sociales del Colegio de Bachilleres y del Colegio de Ciencias y Humanidades. (tesis de maestría). Universidad Nacional Autónoma de México, Ciudad de México.

Oficina Regional de Educación de la Unesco para América Latina y el Caribe [Orealc]. (2008). Los aprendizajes de los estudiantes de América Latina y el Caribe. Primer reporte de los resultados del Segundo Estudio Regional Comparativo y Explicativo. Santiago, Chile: Oficina Regional de Educación de la Unesco para América Latina y el Caribe.

Pila, J. E. (2012). La motivación como estrategias de aprendizaje en el desarrollo de competencias comunicativas de los estudiantes de I-II nivel de inglés del convenio Héroes del Cenepa-Espe de la ciudad de Quito en el año 2012. (tesis de maestría). Universidad de Guayaquil, Ecuador.

Rojas, C. E. (2011). Ética profesional docente: un compromiso pedagógico humanístico. Revista Humanidades: Revista de la Escuela de Estudios Generales, 1(1).

Sacristán, J. (1991). El currículum: una reflexión sobre la práctica. Madrid, España: Editorial Morata.

Sanmartín, N. (2010). Aprender a evaluarse: motor de todo aprendizaje. Aula de Innovación Educativa, 192, 26-69.

Santiago, P., McGregor, I., Nusche, D., Ravela, P. y Toledo, D. (2012). Revisiones de la OCDE sobre la Evaluación en Educación México. México: OCDE / SEP / INEE.

Secretaría de Educación Pública [SEP]. (2011). Plan de estudios. Educación básica. México: Secretaría de Educación Pública.

Secretaría de Educación Pública [SEP]. (2013a). Consejos técnicos escolares. En nuestra escuela... todos aprendemos. Primera sesión ordinaria. México: Secretaría de Educación Pública.

Secretaría de Educación Pública [SEP]. (2013b). La evaluación durante el ciclo escolar. México: Secretaría de Educación Pública.

Secretaría de Educación Pública [SEP]. (2014). Perfil, parámetros e indicadores para docentes y técnicos docentes. México: Secretaría de Educación Pública.

Serrano, R. (2012). La aflicción de la docencia y el tiempo del enseñante. Razón y Palabra, (79). 


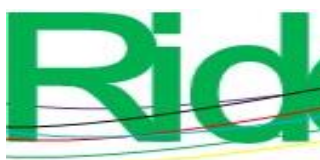

Sikes, P., Measor, L. and Woods, P. (1985). Teacher Career: Crisis and Continuities. London, England: Falmer Press.

Vásquez, D. (2012). Estilos de liderazgo de los docentes del nivel secundaria de una institución educativa del distrito de la Perla-Callao. (tesis de maestría). Universidad San Ignacio de Loyola, Lima. 


\begin{tabular}{|c|c|}
\hline Rol de Contribución & Autor (es) \\
\hline Conceptualización & $\begin{array}{l}\text { Guadalupe Iván Martinez Chairez Principal. Ana Yocihra } \\
\text { Esparza Chavez, Rosa Isela Gómez Castillo (Apoyo) }\end{array}$ \\
\hline Metodología & $\begin{array}{l}\text { Guadalupe Iván Martinez Chairez, Ana Yocihra Esparza } \\
\text { Chavez, Rosa Isela Gómez Castillo (Igual) }\end{array}$ \\
\hline Software & $\begin{array}{l}\text { Guadalupe Iván Martinez Chairez Principal. Ana Yocihra } \\
\text { Esparza Chavez, Rosa Isela Gómez Castillo (Apoyo) }\end{array}$ \\
\hline Validación & $\begin{array}{l}\text { Guadalupe Iván Martinez Chairez, Ana Yocihra Esparza } \\
\text { Chavez, Rosa Isela Gómez Castillo (Igual) }\end{array}$ \\
\hline Análisis Formal & $\begin{array}{l}\text { Guadalupe Iván Martinez Chairez Principal. Ana Yocihra } \\
\text { Esparza Chavez, Rosa Isela Gómez Castillo (Apoyo) }\end{array}$ \\
\hline Investigación & $\begin{array}{l}\text { Guadalupe Iván Martinez Chairez, Ana Yocihra Esparza } \\
\text { Chavez, Rosa Isela Gómez Castillo (Igual) }\end{array}$ \\
\hline Recursos & $\begin{array}{l}\text { Guadalupe Iván Martinez Chairez, Ana Yocihra Esparza } \\
\text { Chavez, Rosa Isela Gómez Castillo (Igual) }\end{array}$ \\
\hline Curación de datos & $\begin{array}{l}\text { Guadalupe Iván Martinez Chairez, Ana Yocihra Esparza } \\
\text { Chavez, Rosa Isela Gómez Castillo (Igual) }\end{array}$ \\
\hline $\begin{array}{l}\text { Escritura - Preparación del } \\
\text { borrador original }\end{array}$ & $\begin{array}{l}\text { Guadalupe Iván Martinez Chairez Principal. Ana Yocihra } \\
\text { Esparza Chavez, Rosa Isela Gómez Castillo (Apoyo) }\end{array}$ \\
\hline $\begin{array}{l}\text { Escritura - Revisión y } \\
\text { edición }\end{array}$ & $\begin{array}{l}\text { Guadalupe Iván Martinez Chairez, Ana Yocihra Esparza } \\
\text { Chavez, Rosa Isela Gómez Castillo (Igual) }\end{array}$ \\
\hline Visualización & $\begin{array}{l}\text { Guadalupe Iván Martinez Chairez, Ana Yocihra Esparza } \\
\text { Chavez, Rosa Isela Gómez Castillo (Igual) }\end{array}$ \\
\hline Supervisión & $\begin{array}{l}\text { Guadalupe Iván Martinez Chairez, Ana Yocihra Esparza } \\
\text { Chavez, Rosa Isela Gómez Castillo (Igual) }\end{array}$ \\
\hline $\begin{array}{l}\text { Administración de } \\
\text { Proyectos }\end{array}$ & $\begin{array}{l}\text { Guadalupe Iván Martinez Chairez, Ana Yocihra Esparza } \\
\text { Chavez, Rosa Isela Gómez Castillo (Igual) }\end{array}$ \\
\hline Adquisición de fondos & $\begin{array}{l}\text { Guadalupe Iván Martinez Chairez, Ana Yocihra Esparza } \\
\text { Chavez, Rosa Isela Gómez Castillo (Igual) }\end{array}$ \\
\hline
\end{tabular}

